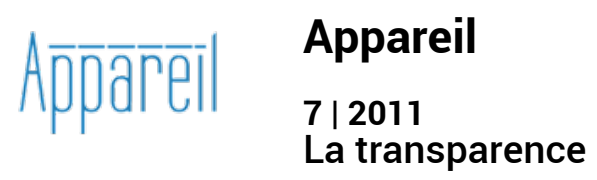

\title{
Nouvelles variations sur la transparence
}

\section{Philippe Junod}

\section{(2) OpenEdition}

Journals

Édition électronique

URL : http://journals.openedition.org/appareil/1197

DOI : 10.4000/appareil. 1197

ISSN : 2101-0714

Éditeur

MSH Paris Nord

Référence électronique

Philippe Junod, « Nouvelles variations sur la transparence », Appareil [En ligne], 7 | 2011, mis en ligne le 11 avril 2011, consulté le 30 juillet 2020. URL : http://journals.openedition.org/appareil/1197 ; DOI : https://doi.org/10.4000/appareil.1197

Ce document a été généré automatiquement le 30 juillet 2020.

\section{(c) (i) (2) $\Theta$}

Appareil est mis à disposition selon les termes de la Licence Creative Commons Attribution - Pas d'Utilisation Commerciale - Pas de Modification 4.0 International. 


\title{
Nouvelles variations sur la transparence
}

\author{
Philippe Junod
}

\section{NOTE DE L'AUTEUR}

Une première version de cet essai a paru dans le catalogue de l'exposition du Musée national de Monaco : Jean-Michel Bouhours (dir.), Lumière, transparence, opacité, Milano, Skira, 2006, p. 154-159.

1 C'est qu'il y a transparence et transparence. Littérale ou phénoménale, réelle ou virtuelle, selon les termes de Colin Rowe et Robert Slutzky, dont l'étude a fait l'objet de réactions et commentaires divers ${ }^{1}$.

Commençons par la première, l'optique. Le verre en est l'incarnation par excellence, comme matériau ou medium, voire en tant que sujet d'une représentation (que l'on pense à la célèbre nature morte de Sébastien Stoskopff au Musée de Strasbourg). Pratiqué dès l'antiquité, illustré au Moyen Âge par l'essor du vitrail, relancé par l'Art nouveau, Gallé, Daum ou Tiffany ${ }^{2}$, l'art des maîtres verriers connaîtra un succès croissant jusqu'à nos jours, comme en témoignent nombre d'expositions ou de publications récentes ${ }^{3}$.

3 La peinture sous verre, d'origine populaire, fut pratiquée par quelques maîtres modernes, dont Kandinsky. Quant aux «transparents », ce sont des toiles translucides, peintes sur les deux faces, qu'un éclairage placé alternativement devant ou derrière permet d'animer. Ce procédé, très en vogue aux XVIII ${ }^{e}$ et XIX ${ }^{e}$ siècles, suscite aujourd'hui un regain d'intérêt ${ }^{4}$. À l'eidophysikon de Loutherbourg succèdera le diorama de Daguerre. C'est aussi la perméabilité des supports à la lumière qui est à l'origine de la photographie, de Niepce à Talbot, et du négatif à la diapositive. Pour ce qui est de la projection, de la lanterne magique au cinéma, la transparence matérielle de la plaque ou de la pellicule se réplique dans celle, virtuelle, de l'écran ${ }^{5}$. On ne saurait non plus oublier le cliché-verre cher à $\operatorname{Corot}^{6}$. La vocation poétique de la plaque sensible 
s'affirmera encore avec les photogrammes, rayogrammes, doubles expositions et autres procédés de surimpression ${ }^{7}$, sans parler de la radiographie (voir l'autoportrait aux rayons X de Meret Oppenheim, 1964).

4 La sculpture, au $\mathrm{xx}^{\mathrm{e}}$ siècle, est également fascinée par la transparence, tant dans le choix des matériaux que par le jeu des formes virtuelles, des constructions de Gabo et Pevsner aux vides qui articulent les figures de Henry Moore, sans parler de l'op art. Quant aux ambiguïtés spatiales du "modulateur espace-lumière » de Moholy-Nagy, elles prolongent ses expériences sur la superposition des surfaces qui caractérise ses tableaux exécutés au Bauhaus. Et si les light drawings d'Anne Blanchet jouent de la translucidité du plexiglas pénétré par les rayons lumineux, son installation réalisée en 1997 au Forum d'art contemporain de Sierre, intitulée PORTES 97, musique visuelle, multiplie les transparences en disposant l'une derrière l'autre des portes vitrées coulissantes (fig. 1$)^{8}$.

Emblème de la modernité industrielle et architecturale (fig. 2), le verre, enchâssé dans une structure métallique, fut d'abord utilisé pour les serres. Consacré par le succès du Crystal Palace de Joseph Paxton à l'exposition universelle de Londres en 1851, le procédé allait bientôt abriter marchés, halles d'expositions, grands magasins, passages couverts (fig. 3 et 4) et gares ferroviaires'. Ouverture de l'espace, hymne à la lumière définiront bientôt le nouveau style "international ", du Bauhaus aux gratte-ciels contemporains (fig. 5 et 6), où le jeu des reflets instaure un autre type de transparence (fig. 7 à 9) ${ }^{10}$. Par ailleurs, prônée dès $1898^{11}$, la maison de verre se voyait conférer par Paul Scheerbart une dimension allégorique ${ }^{12}$, et le mouvement de la Gläserne Kette devait s'en inspirer. Bruno Taut (Pavillon du Werkbund à Cologne, 1914), Mies van der Rohe (Pavillon de Barcelone, 1929), Le Corbusier (Pavillon suisse de la Cité universitaire à Paris, 1933), Philip Johnson (Glass House à New Canaan, dans le Connecticut,1949), Peter Zumthor (Musée de Bregenz, 1991) ou Takashi Yamagushi (Temple du verre à Kyoto, 2000), pour n'en citer que quelques-uns, confèreront ses lettres de noblesse à une esthétique où se croisent des composantes utopiques, oniriques, mystiques, hygiénistes, publicitaires, idéologiques, morales, politiques ou sociales ${ }^{13}$. Pierre Chareau, dans sa Maison de verre à Paris (1928-1932), poussera la transparence jusqu'à utiliser des panneaux de tôle perforées pour des cloisons intérieures ${ }^{14}$. Banalisée de nos jours, l'architecture de verre sera souvent remise en question ${ }^{15}$.

Mais il est une autre transparence, d'ordre stylistique, celle qu'a définie Colin Rowe à propos de la Villa construite par Le Corbusier à Garche. Dans son commentaire paru quelques années plus tard, Bernhard Hoesli généralisait cette catégorie critique pour l'appliquer à toute l'histoire de l'architecture, de Leon Battista Alberti à Frank Lloyd Wright ${ }^{16}$. On rappellera que Rowe était un élève de Rudolph Wittkower, qui avait luimême pratiqué ce genre d'approche phénoménologique à propos de Palladio, Rainaldi et Longhena, et proposé le concept de "principe d'ambiguïté ${ }^{17}$ ». Cependant c'est sur l'examen d'un tableau de Fernand Léger que se fondait l'analyse formelle de Rowe, et R. Slutzky était un disciple d'Albers. Une étude plus récente a d'ailleurs repris l'étude du cubisme sous cet angle ${ }^{18}$.

7 Certes, la peinture relève d'abord de la transparence littérale. Glacis, aquarelle, aérographe, grattage, palimpseste sont ici autant de procédés et de techniques qui 
laissent transparaître le fond et favorisent la superposition et l'échelonnement des plans en profondeur. On sait d'autre part que la fenêtre ouverte est un motif récurrent dans l'iconographie occidentale, et que la perspective est liée étymologiquement à la transparence, puisque perspicere signifie "voir à travers ». La démonstration première en est fournie par la fenêtre ou graticola d'Alberti, la pariete di vetro de Léonard ou le portillon de Dürer, dispositifs qui se prolongeront jusqu'au "perspecteur » de Van $\mathrm{Gogh}^{19}$, voire à la quatrième paroi, invisible, de la scène du théâtre naturaliste.

En fait, ce paradigme survivra parfois à la crise de l'espace figuratif. Car si la transparence de la toile ou du mur peut sembler contrariée par la fragmentation de la surface picturale, du divisionnisme néo-impressionniste au cubisme analytique, et jusqu'aux photomontages de David Hockney, ces fausses opacités sont en fait des grilles plaquées sur un spectacle dont la syntaxe reste souvent conventionnelle. On peut en dire autant des superpositions de Picabia. La fenêtre d'Alberti, revue et corrigée par celles de Delaunay (1912-1914), connait ainsi une deuxième carrière, et Boccioni pourra associer transparence et simultanéité sous la rubrique de la "compénétration des plans $^{20} »$. D'autre part, la vitre, mise en abyme, a fait l'objet d'un procès insidieux chez Magritte, qui joue la carte du « détrompe l'œil », de La condition humaine aux Promenades d'Euclide, tandis que Duchamp manie la preuve par l'absurde avec Fresh Widow, ou la Bagarre d'Austerlitz. Quant au paradoxe de la transparence perverse de son Grand verre, dont l'hermétisme peut apparaitre comme le comble de l'opacité, on sait qu'il entretient avec la théorie perspective des liens dialectiques ${ }^{21}$. Les surréalistes, enfin, ont su jouer des ambiguïtés du reflet et de l'ombre, deux corollaires de la transparence liés aux origines mythologiques de la peinture (le miroir de Narcisse et le mur de Dibutade).

9 Mais on connaît encore d'autres transparences. Iconographique par exemple, avec l'«architecture parlante» d'un Claude-Nicolas Ledoux. Quant à la transparence fonctionnelle, celle qui détermine la forme dans le design, on mesure son importance en architecture, où la structure intérieure peut se révéler dans l'enveloppe, la façade par exemple. Poussée à bout dans les fameuses "montres-squelettes », dont le mécanisme est comme mis en vitrine dans une boîte de verre, elle est également au principe du traité de Physiognomonie de Lavater (dont l'illustration par silhouettes fait d'ailleurs un large usage du physionotrace, un lointain dérivé de la fenêtre albertienne) : "Que l'on n'oublie pas un instant que les caractères extérieurs n'existent que pour faire connaître l'intérieur !22 "

10 Il est aussi des transparences horizontales, comme celle du paragone, qui postule la correspondance, et donc la traductibilité d'un art à l'autre. C'est ainsi que la doctrine classique de l'ut pictura poesis affirme la présence du texte derrière l'image, et vice versa. Sa version musicaliste, d'obédience synesthésique et souvent inspirée par le principe de l'audition colorée, connaîtra une fortune croissante au $\mathrm{xx}^{\mathrm{e}}$ siècle $^{23}$. Paul Klee, dont les "polyphonies» sont traitées par stratifications chromatiques ou texturales, en est le représentant le plus connu.

11 Les transparences verticales sont d'ordre métaphysique (le macrocosme derrière le microcosme, la quatrième dimension derrière la troisième) ou mystiques (la transcendance). C'est la dialectique symboliste du voile qui, comme dans sa version érotique, cache et révèle tout à la fois. On sait l'influence des courants occultistes sur les avant-gardes, Kandinsky, Mondrian, Kupka, Malevitch, Duchamp, entre autres ${ }^{24}$. La transparence ésotérique s'affirmera jusque chez Breton, dont une célèbre déclaration 
inverse la métaphore albertienne : «C'est ainsi qu'il m'est impossible de considérer un tableau autrement que comme une fenêtre dont mon premier souci est de savoir sur quoi elle donne...25 » On peut rappeler de même, à propos de "l'art magique surréaliste ", cette formule symptomatique : «Pour ouvrir à volonté sa fenêtre sur les plus beaux paysages du monde et d'ailleurs ${ }^{26}$. " En 1942, le pape du surréalisme déclarait encore vouloir "ouvrir les fenêtres sur les plus grands paysages utopiques ", et invoquait « Les grands transparents », qualifiés de « mythe nouveau ${ }^{27}$ ».

La fascination du cristal, chez J. J. Rousseau ou Le Corbusier par exemple ${ }^{28}$, témoigne de l'importance du réseau métaphorique qui entoure la transparence. Aussi polyvalente qu'omniprésente, la notion a trouvé preneur dans les domaines les plus divers. Invoquée en littérature, tant dans la création romanesque ${ }^{29}$ que par la critique ${ }^{30}$, à propos de Rousseau, Musset ${ }^{31}$, Zola $^{32}$ ou Breton ${ }^{33}$, on la retrouve en ophtalmologie ${ }^{34}$, philosophie ${ }^{35}$, sociologie ${ }^{36}$, en linguistique avec les théories mimétiques sur l'origine du langage ${ }^{37}$, puis en sémiotique ou poétique ${ }^{38}$. En faisant de La Mariée de Duchamp une " peinture d'idées » et un "mythe de la critique », Octavio Paz lui confère une valeur épistémologique ${ }^{39}$. Et de la transparence sémantique, ou du signifié derrière le signe ${ }^{40}$, résulte sa version esthétique, celle de l'intention délibérée, du dessein derrière le dessin, ou du « coin de la création vu à travers un tempérament » de Zola ${ }^{41}$. Autour du phénomène optique se profilent ainsi divers sens figurés, telles les acceptions morale ou psychologique ${ }^{42}$, médicale, politique (la glasnost), juridique, administrative, économique, publicitaire, etc. ${ }^{43}$. Bref, la transparence est devenue un slogan passepartout ${ }^{44}$. Tel est le prestige d'une notion dont la définition transitive s'enrichit de connotations flatteuses: luminosité, clarté, lucidité, évidence, franchise, honnêteté, pureté, limpidité, et ainsi de suite, qualités dont l'opacité apparaît alors comme la privation. Ainsi Breton, s'insurgeant contre «la littérature psychologique à affabulation romanesque ", pouvait-il reprendre la métaphore chère aux architectes : « Pour moi, je continuerai à habiter ma maison de verre [...] où je repose la nuit sur un lit de verre aux draps de verre...45 "Et Susan Sontag, dans son essai Contre l'interprétation, qui critique pourtant l'idée de "contenu» et prône l'attention à la forme, en arrive à écrire que "la transparence est aujourd'hui la plus haute valeur libératrice en matière d'art comme de critique. Elle consiste à éprouver la luminosité de la chose elle-même ${ }^{46}$.»

Tout comme la perspective, la transparence est marquée du sceau de l'ambivalence. En effet, la construction albertienne, qui s'était d'abord définie comme un instrument de précision destiné à mesurer ou à reproduire le monde visible, n'allait pas tarder à se mettre au service de l'irrationnel et de la subjectivité, par la pratique de l'anamorphose notamment. Il en va de même de la transparence, symbole de l'univocité, qui devait connaître des usages équivoques et fournir un outil privilégié aux ambiguïtés spatiales cultivées par les avant-gardes constructivistes, chez Moholy-Nogy ou Lissitzky en particulier. Dans les deux cas également, une donnée "naturelle» et objective s'enrichit d'une dimension culturelle : le technique et le mental sont ici les deux faces d'une même entité. La transparence, comme la perspective, a donc une histoire, et l'on pourrait parler à son propos de «forme symbolique » au sens où l'entendait Panofsky ${ }^{47}$. 
L'histoire des arts montre que l'optique ne coïncide pas nécessairement avec l'esthétique. Dans une étude parue il y a une trentaine d'années, j'avais assimilé l'opacité à la modernitét8. Louis Marin a depuis inversé la métaphore ${ }^{49}$, et Rosalind Krauss l'a réfutée ${ }^{50}$. D'autre part, on peut se demander si la transparence n'a pas opéré divers retours dans l'art moderne et contemporain, où les matériaux diaphanes ou translucides connaissent un succès croissant, du vitrail (qui inspira Kupka comme Delaunay) au gemmail, du verre (Marcel Duchamp) au plexiglas (Gabo et Pevsner, Georges Vantongerloo) ou au plastique (Piero Manzoni), sans parler de l'eau (Gyula Kosice, Emmanuel Saulnier), de l'air (encore Duchamp, Yves Klein), du gaz, etc. Par ailleurs, on connaît l'importance renouvelée de la photographie en peinture, des hyperréalistes américains jusqu'à Gerhard Richter. Et que dire des Transparencies de Jeff Wall, pour qui la projection de "l'image luminescente» constitue "une forme essentielle de l'expérience de la modernité ${ }^{51}$ "? De l'hologramme au laser ou à l'image numérisée, de la vidéo à la color music, l'art s'est en effet souvent dématérialisés5. F. Kupka envisageait déjà une création «sans aucune médiation matérielle ${ }^{53}$ ». Et la disparition de l'œuvre au sens traditionnel, dans les happenings ou performances par exemple, va dans le même sens. L'art conceptuel n'est-il pas le lieu d'une prédominance de l'intention sur la réalisation, celle-ci pouvant, à la limite, se révéler facultative ? Et qu'y a-t-il de plus transparent que les silences de John Cage?

\section{Figures}

Figure 1

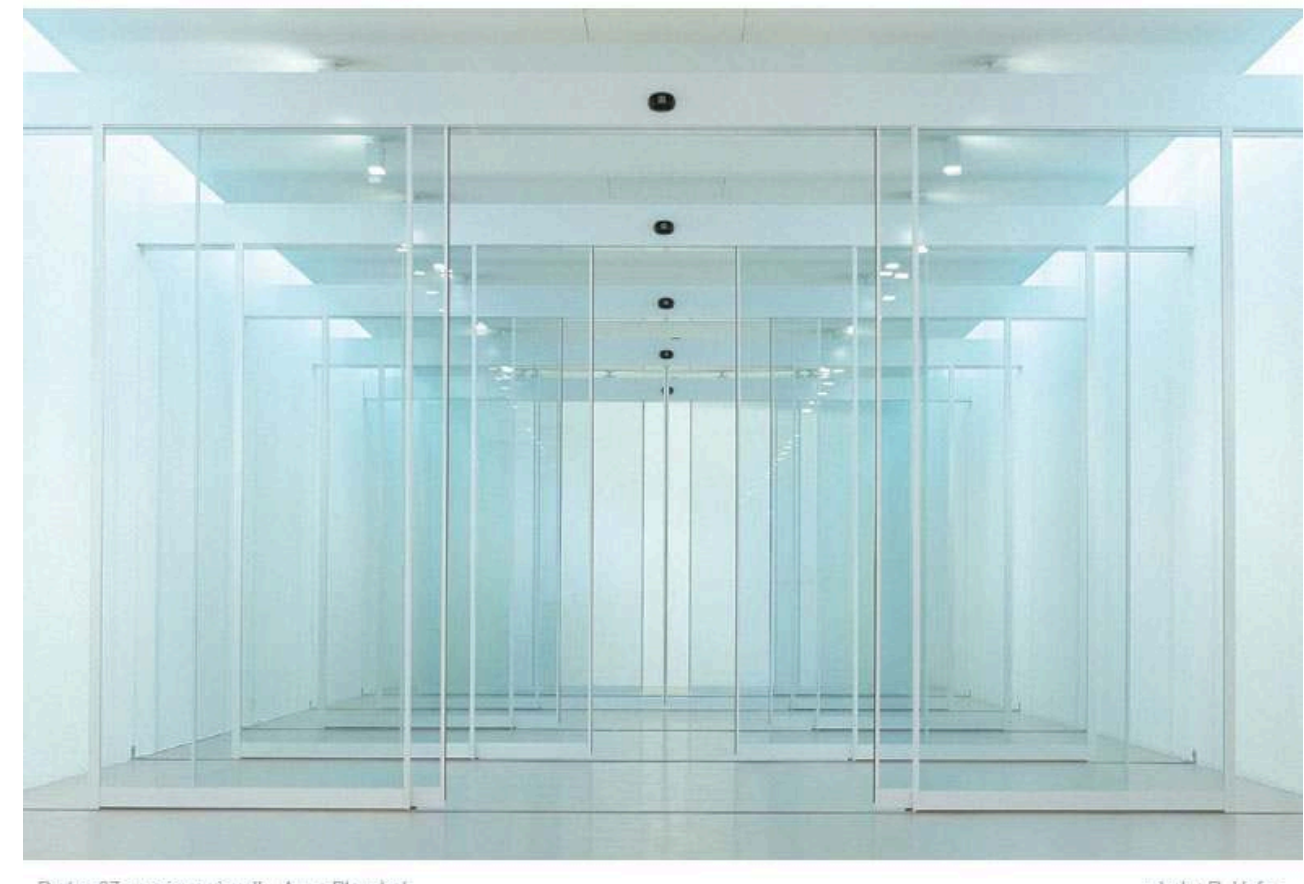

Portes 97, musique visuelle. Anne Blanchet

photo: R. Hofer

PORTES 97, musique visuelle

copyright Anne Blanchet 
Figure 2

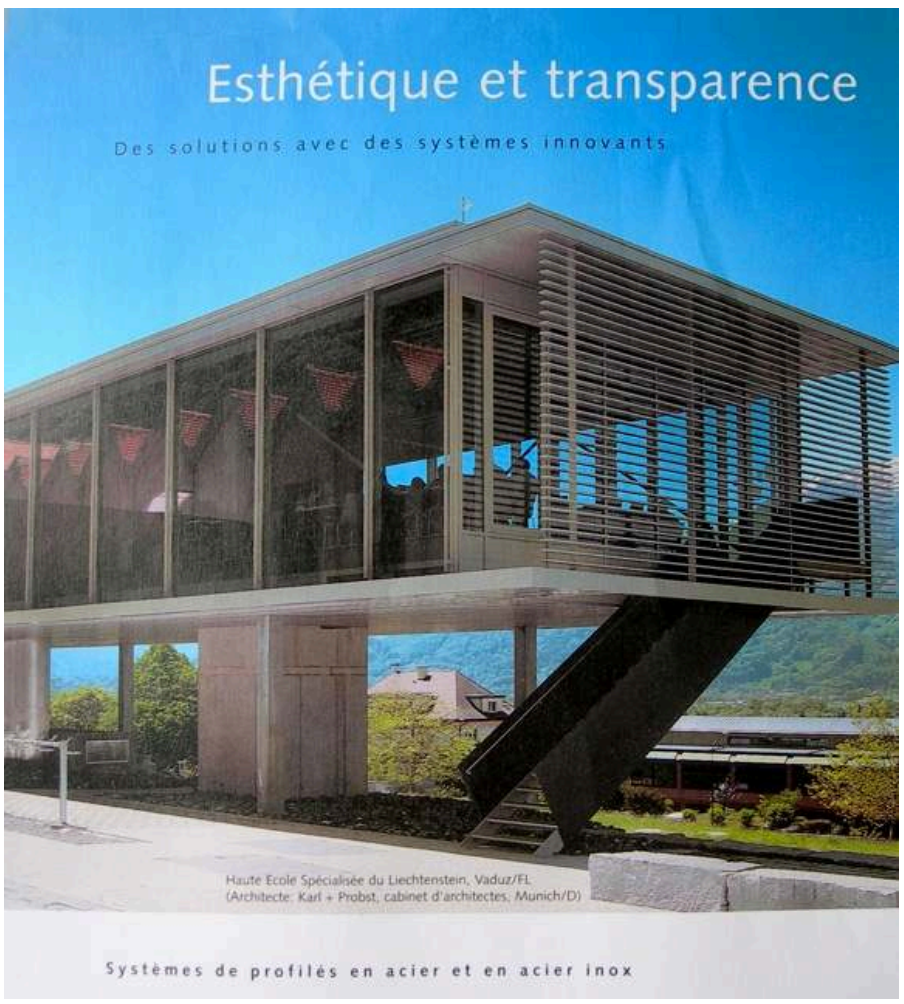

Publicité de la Haute École spécialisée du Liechtenstein, Vaduz

Figure 3

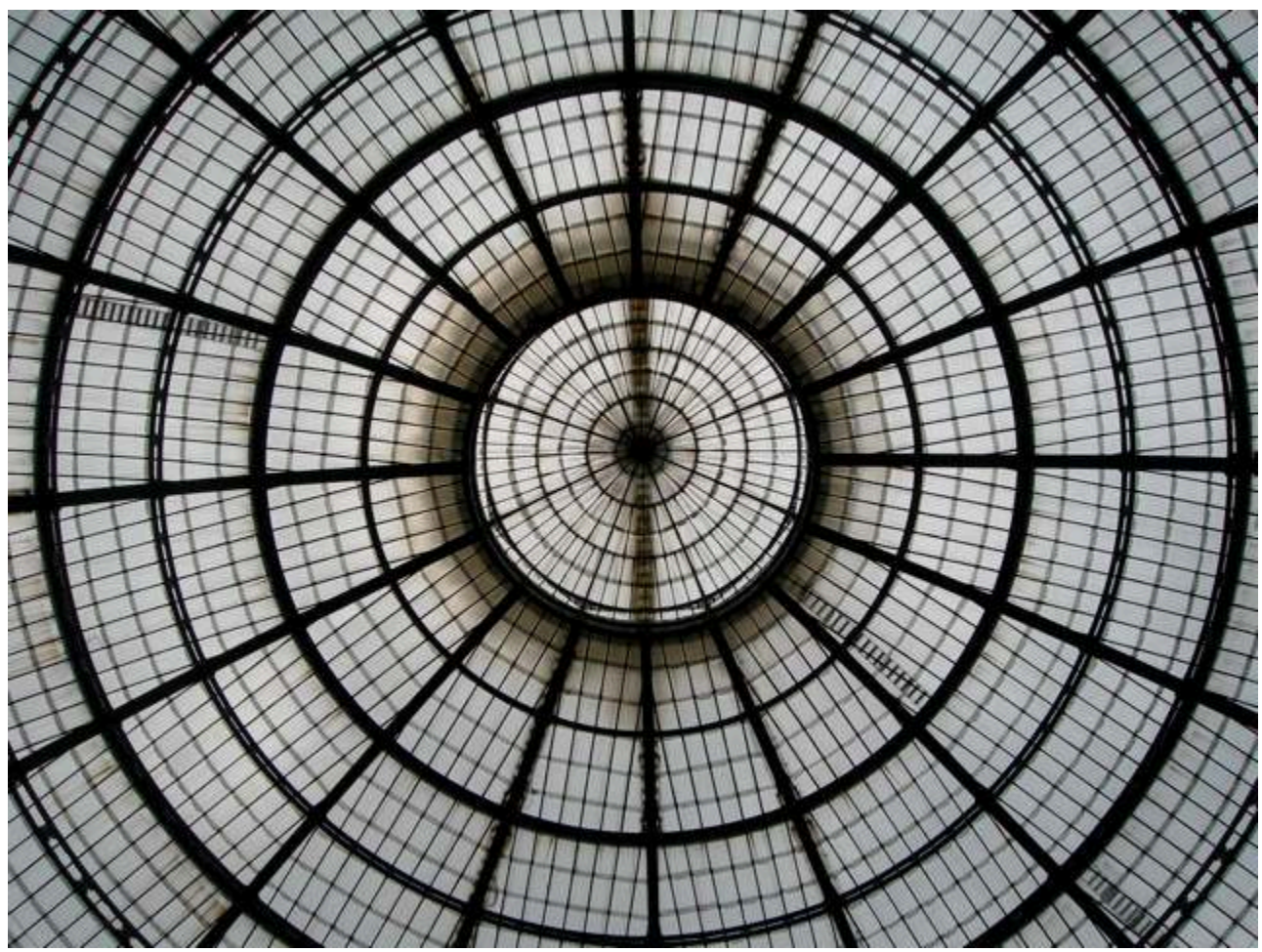

Milan, Galerie Vittorio Emmanuele II, 1865-1867, architecte. G. Menagoni 
Figure 4

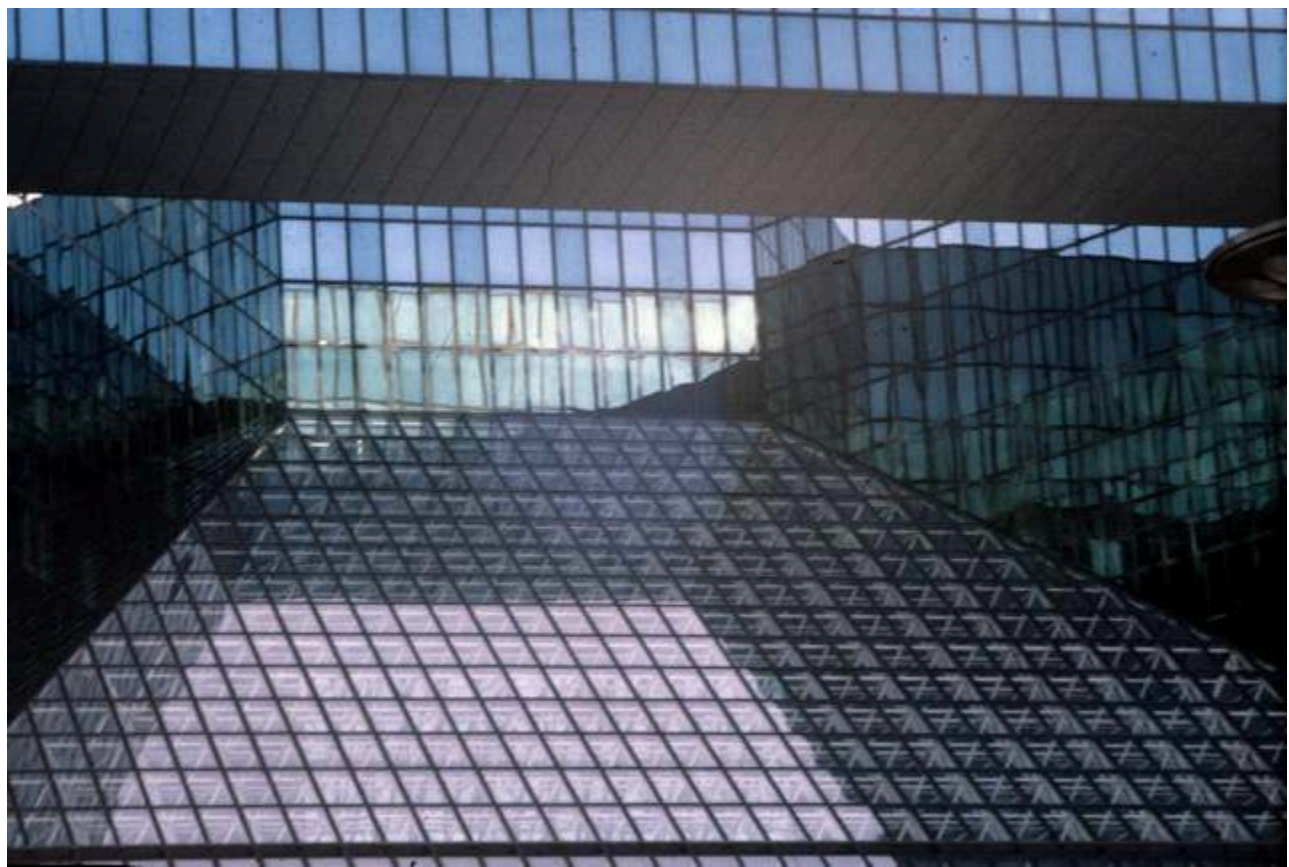

Paris, La Défense

Figure 5

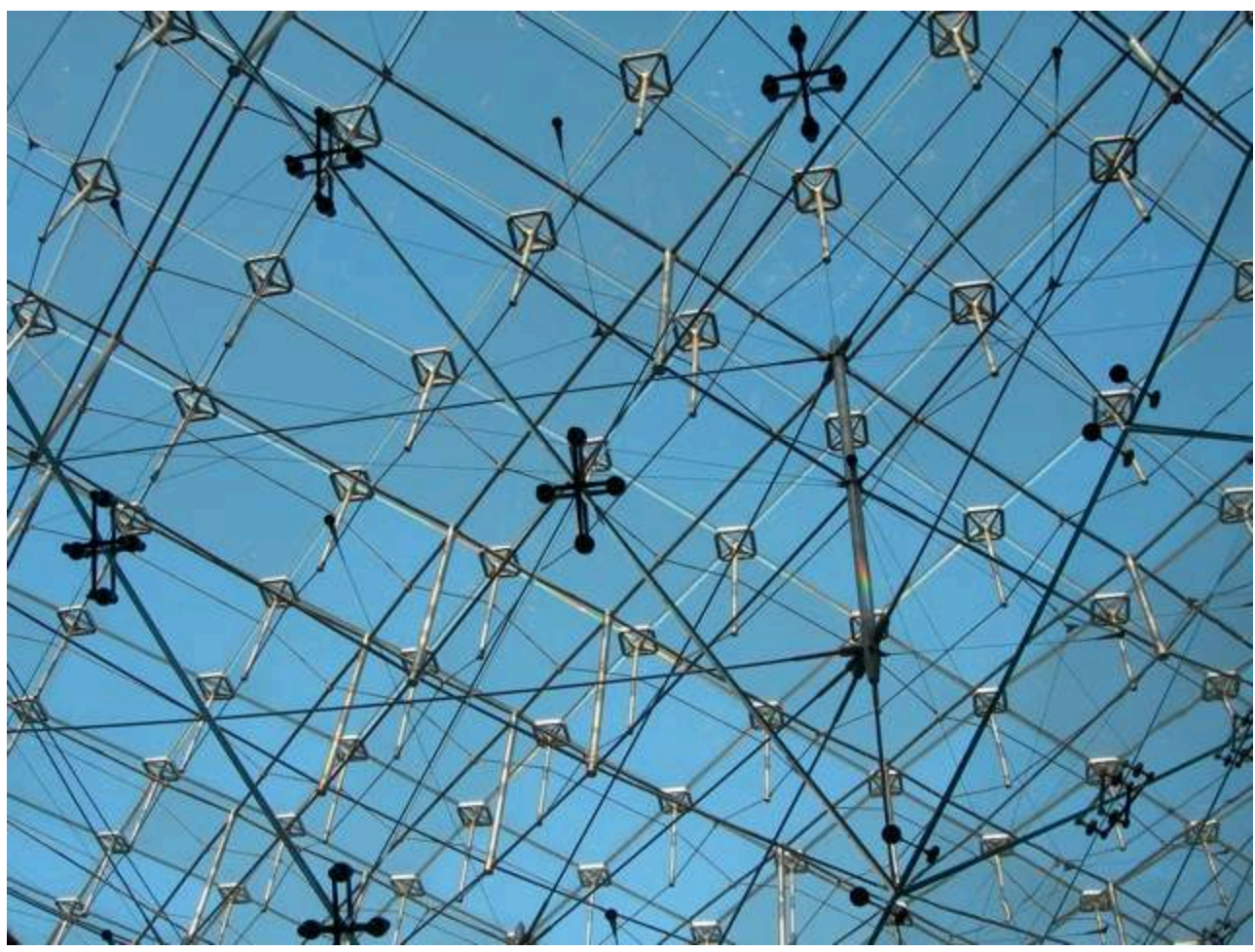

Pyramide de Pei au Louvre, Paris 
Figure 6

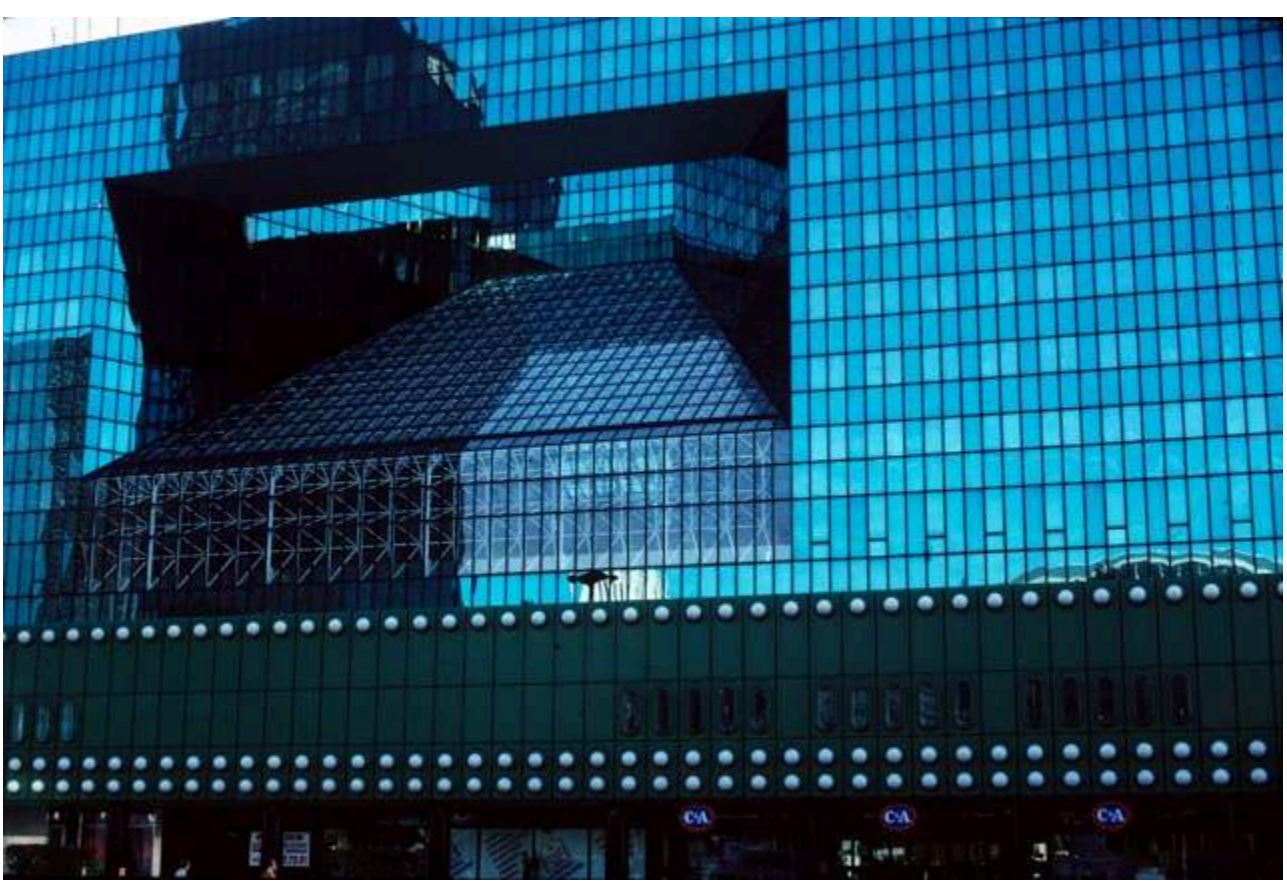

Paris, La Défense

Figure 7

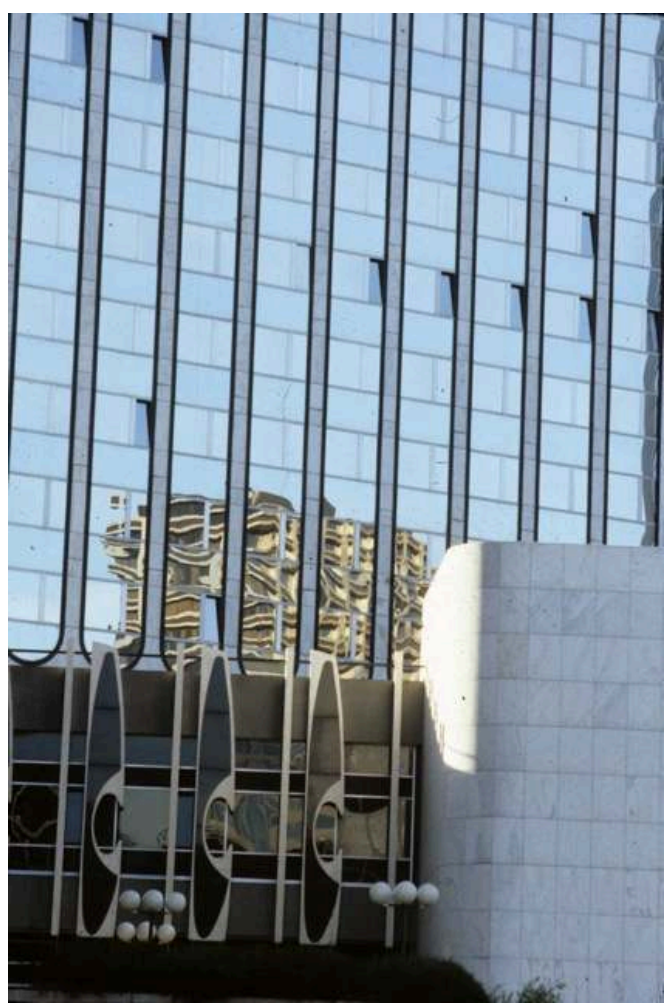

Paris, La Défense 
Figure 8

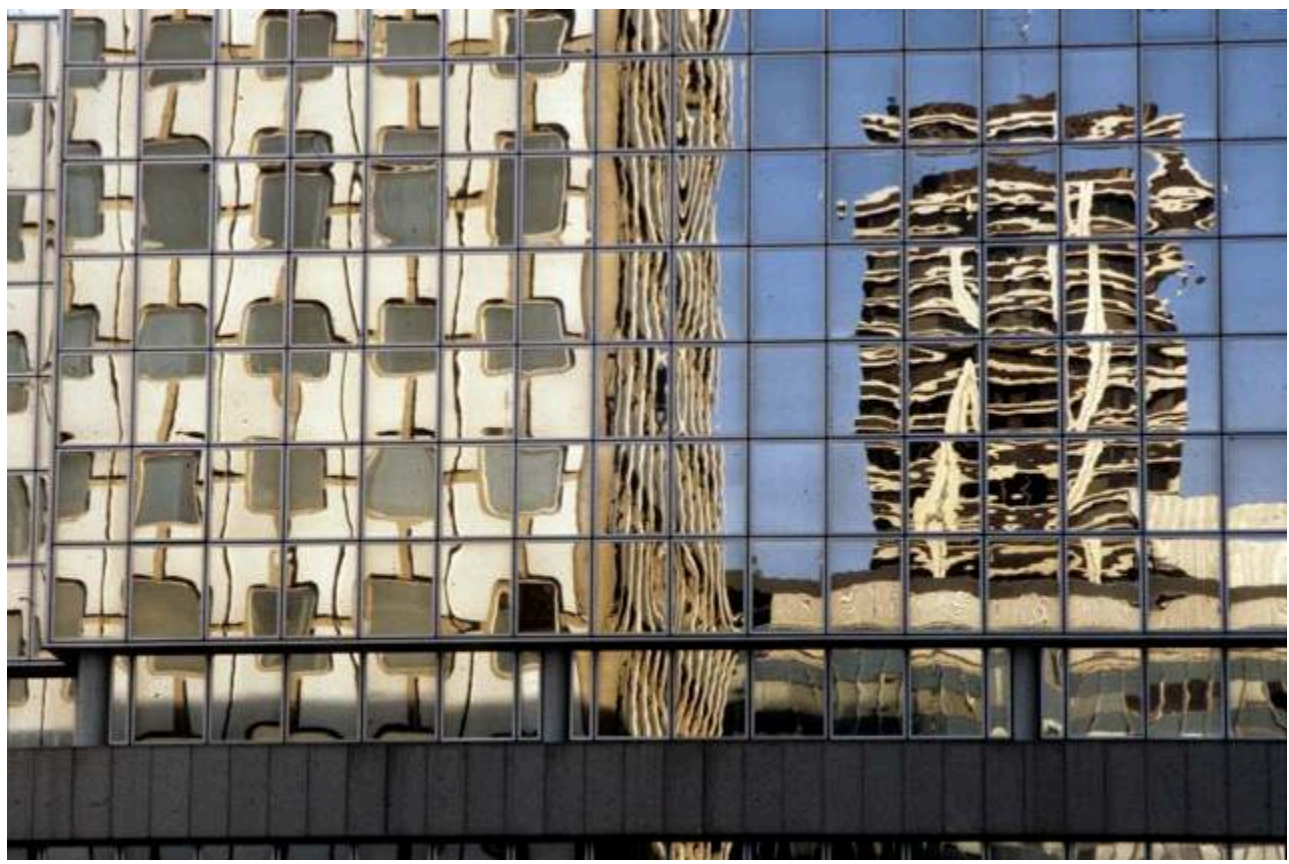

Paris, La Défense

Figure 9

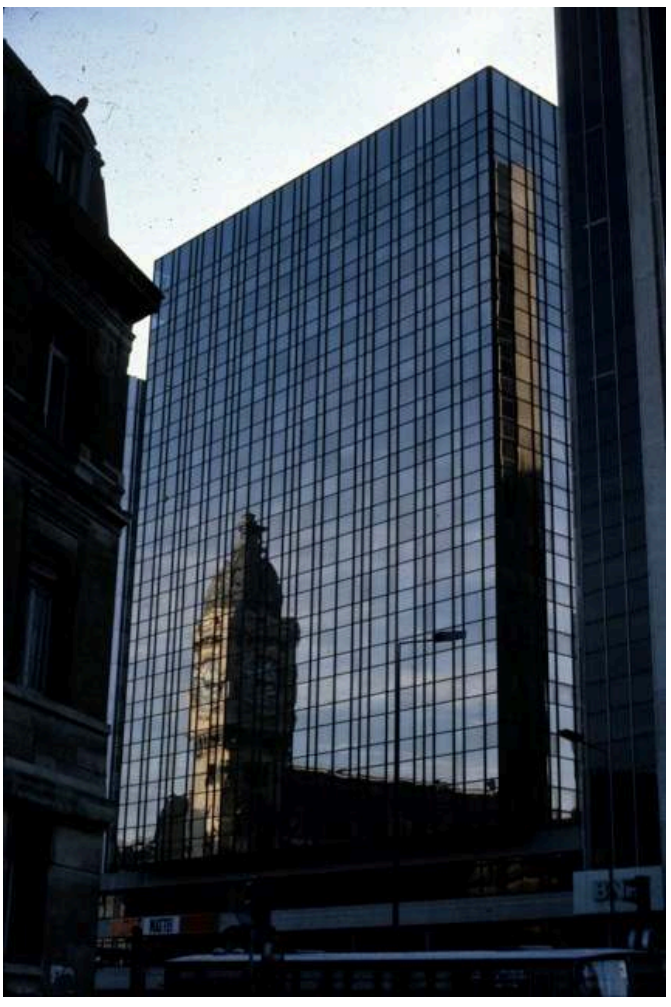

Paris, gare de Lyon 


\section{NOTES}

1. Colin Rowe / Robert Slutzky, "Transparency, Literal and Phenomenal", Perspecta, Yale Architectural Journal, VIII, 1963, et XIII/XIV, 1971, trad. Transparenz, 1968, rééd. Basel, Birkhäuser, Zürich: ETH, 1974 [Le Corbusier Studien 1] et 1989, et Transparence réelle et virtuelle, Paris, Demicercle, 1992. Pour des commentaires critiques, cf. Stanislaus von Moos, Revue suisse d'art et d'archéologie, 27, 1970, p. 237-38; Werner Oechslin, "Transparence, ou à la recherche d'une méthode de projet basée sur les principes de l'architecture moderne ", introduction à la traduction française citée, et Rosemarie Haag-Bletter, "Opaque Transparency”, Oppositions, 13, 1978, p. 121-26.

2. Le verre Art nouveau, Gingins, Fondation Neumann, 1994 ; Helmut Ricke / Eva Schmitt (éd.), Art Nouveau Glass. The Gerda Koepff Collection, London, Prestel, 2004 ; Tiffany \& Gallé : Art Nouveau Glass, Budapest, Museum of Applied Arts, 2007 ; Rosalind Pepali de Mestral, Louis C. Tiffany : le maître du verre, Paris, Gallimard, 2009.

3. Voir entre autres Olga Drahotova, L'art du verre en Europe, Paris, Gründ, 1984 ; Marina Causa, L'art du verre de la Renaissance à nos jours, Paris, Payot, 1987 ; Transparence. L'art du verre en France de 1914 à 1960, Orléans, Musée des Beaux-Arts, 1987 ; Florian Lechner, «Une collection de verre unique en Europe... au Musée des Arts décoratifs de Lausanne ", Architecture romande, 1991, nº 2, p. 79-80 ; Catherine Vaudout, L'art du verre contemporain, Paris, A.Colin, 1992 ; Rosa Barovier Mentasti, Les âges du verre. Histoire et techniques du verre de l'Antiquité à nos jours, Milan, Skira, 2003 ; Jacqueline Bellanger, Histoire du verre. L'aube des temps modernes, 1453-1672, Paris, Massin, 2006 ; Merveilles du verre. Trésors des musées et des collections privées d'Alsace, de l'Antiquité à l'Art nouveau, Colmar, Musée d'Unterlinden, 2006. Sur les propriétés optiques du verre, voir aussi Libero Zuppiroli et al., Traité de la lumière, Lausanne, Presses polytechniques universitaires romandes, 2009, ch. 9.

4. Cf. le catalogue de Monaco, note 1.

5. Wheeler Winston Dixon, The Transparency of Spectable: Meditations on tne Moving Image, Albany, State Univ. of New York Press, 1988 ; Rebecca M. Pauly, The Transparent Illusion: Image and Ideology in French Text and Film, New York, Lang, 1993; François Albèra, «Destruction de la forme et transparence au cinema », Cahiers du Musée national d'Art Moderne, $n^{\circ}$ 48, été 1994, p. 49-63.

6. Rainer Michael Mason, Le cliché-verre. Corot et la gravure diaphane, Genève, Cabinet des estampes, 1982.

7. Alain Fleig, "Zielke et la transparence des choses ", Cahiers de la photographie, $\mathrm{n}^{\circ}$ 8, 1982, p. 91-99; Ursula Czartoryska, «Transparence. Un saut à travers la pellicule. L'entrée dans un autre monde ", in La transparence dans l'art du Xxe siècle, Le Havre, musée des Beaux-Arts / A. Biro, 1995, p. 75-98.

8. En mouvement dans le DVD d'Anne Blanchet, Musiques visuelles 1997-2000, Prix Gustave Buchet 2000.

9. Monica Henning-Schefold / Helga Schmidt-Thomsen, Transparenz und Masse. Passagen und Hallen aus Eisen und Glas, Köln, DuMont, 1972 ; Georg Kohlmaier / Barna von Sartory, Das Glashaus, ein Bautypus des 19. Jh., München, Prestel, 1981; Ruth-Maria Ullrich, Glas-Eisenarchitektur. Pflanzenhäuser des 19. Jh., Worms, Werner, 1989. Voir aussi les contributions de Thierry Paquot et Marc Perelman dans Pascale Dubus (éd.), Transparences, Paris, Éd. de la Passion, 1999, p. 101-119 et 121-142.

10. Transparences, numéro spécial de la revue Faces. Journal d'architectures, ${ }^{\circ} 45$, hiver 1998-1999 ; Christian Schittich et al., Construire en verre, Lausanne, Presses polytechniques et universitaires romandes, 2001 ; Bernard Tschumi et al., Glass Ramps / Glass Wall. Deviations from the Normative, 
London, Architectural Association, 2001 ; Brent Richards, Nouvelle architecture de verre, Paris, Seuil, 2006.

11. Jules Henrivaux, "Une maison de verre ", Revue des deux Mondes, CL, $1^{\text {er }}$ novembre 1898 , p. 112-139.

12. Paul Scheerbart, Glas Architektur (1914), trad. L'architecture du verre, Strasbourg, Circé, 1995.

13. Voir à ce sujet Emmanuel Alloa, "Architectures of Transparency", Re. Anthropology and Aesthetics, 53/54, Spring/autumn 2008, p. 322-330.

14. Brian Brace Taylor, Pierre Chareau, Designer and Architect, Köln, Taschen, 1998, p. 104-149; Dominique Vellay, La maison de verre, le chef d'oeuvre de Pierre Chareau, Arles, Actes Sud, 2007.

15. Alan Colquhoun, "Plateau Beaubourg", Architectural Design, XLVII, 1977, $n^{\circ} 2$, rééd, in Collected Essays in Architectural Criticism, London, Black Dog, 2009, p. 82-89 ; Claude Parent, « Vous avez dit transparence? ", L'architecture d'aujourd'hui, n² 269, juin 1990, p. 24-26.

16. Bernhard Hoesli, "Kommentar", in Rowe / Slutzky, Transparenz, note 2, p. 49 sq. Pour un autre exemple d'utilisation de cette catégorie critique, $c f$. Pascal Lorenz / Joël Chervaz, « Transparence et opacité architecturales relevées dans l'étude du bâtiment viennois du Palais équitable", Cahiers d'enseignement et de recherches, 1, Lausanne, EPFL, Département d'architecture, 1971, p. 10-58.

17. Rudolph Wittkower, "Carlo Rainaldi and the Architecture of the High Baroque in Rome" [1937], in Studies in the Italian Baroque, London, Thames \& Hudson, 1975, p. 9-52 (46), et "Santa Maria della Salute" (1963), Ibid., p. 125-152.

18. Nicolaj van der Meulen, Transparente Zeit. Zur Temporalität kubistischer Bilder, München, Fink, 2002.

19. Van Gogh, Correspondance, Paris, Gallimard, 1990, I, p. 670-671.

20. Umberto Boccioni, Dynamisme plastique [1914], éd. G. Lista, Lausanne, L’Âge d'Homme, 1973, p. 82 .

21. Jean Clair, «Marcel Duchamp et la tradition des perspecteurs ", in L'œuvre de M. Duchamp. Abécédaire, Paris, Musée national d'art moderne, 1977, p. 124-65.

22. Johann Caspar Lavater, Physiognomische Fragmente (1775-78), trad. La physiognomonie, ou l'art de connaître les hommes, Lausanne, L'Âge d'homme, 1979, p. 26.

23. Philippe Junod, « De l'audition colorée ou du bon usage d'un mythe », in Contrepoints. Dialogues entre musique et peinture, Genève, Contrechamps, 2006, p. 67-106.

24. Maurice Tuchman (éd.), The Spiritual in Art: Abstract Painting 1890-1985, Los Angeles, County Museum of Art, 1986 ; Christoph Wagner (éd.), Das Bauhaus und die Esoterik. J. Itten, W. Kandinsky, P. Klee, Würzburg, Museum im Kulturspeicher / Kerber Verlag, 2006.

25. André Breton, Le surréalisme et la peinture (1928), Paris, Gallimard, 1965, p. 2.

26. Id., « Oscar Dominguez » (1936), Ibid., p. 129.

27. Id., "Prolégomènes à un troisième manifeste du surréalisme » (1942), in Manifestes du surréalisme, Paris, Gallimard (Idées), 1970, p. 174-176.

28. Jean Starobinski, «La transparence du cristal », in J. J. Rousseau. La transparence et l'obstacle, Paris, Plon, 1957, p. 317 sq. ; Amédée Ozenfant / Charles Edouard Jeanneret, « Vers le cristal», Esprit nouveau, $\mathrm{n}^{\circ} 25$, juillet 1924.

29. Par exemple Vassily Photiades, Les transparents, Paris, Stock, 1958, ou Vladimir Nabokov, Transparent Things [1972], trad. La transparence des choses, Paris, Fayard, 1979.

30. Dorrit C.Cohn, Transparent Minds. Narrative Modes for Presenting Consciousness in Fiction, Princeton: Univ. Press, 1978, trad. La transparence intérieure: modes de représentation de la vie psychique dans le roman, Paris, Seuil, 1981 ; Pierre Brunel, Transparence du roman. Le romancier et ses doubles au $\mathrm{XX}^{e}$ siècle, Paris, Corti, 1997.

31. Alain Heyvaert, La transparence et l'indicible dans l'œuvre d'Alfred de Musset, Paris, Klincksieck, 1994. 
32. Philippe Hamon, «Zola romancier de la transparence ", Europe, $n^{\circ} 468-469$, avril-mai 1968, p. 385-391.

33. Michel Beaujour, "André Breton ou la transparence », in André Breton, Arcane 17, Paris, Union générale d'éditions [10/18], 1965, p. 161-83.

34. Yves Pouliquen, La transparence de l'œil, Paris, Odile Jacob, 1992.

35. Anca Vasiliu, Du diaphane. Image, milieu, lumière dans la pensée antique et médiévale, Paris, Vrin, 1997, et «Le transparent, le diaphane et l'image ", in P. Dubus, note 10, p. 15-29 (à propos d'Aristote). Voir aussi la critique de la «théorie de la transparence» par Arthur Danto, The Transfiguration of the Common Place, Cambridge, Harvard Univ. Press, 1981, p. 156-64, et The Philosophical Disenfranchisement of Art [1986], trad. L'assujettissement philosophique de l'art, Paris, Seuil, 1993, p. 48 sq.

36. Alain Finkielkraut, "Le devoir de transparence», Temps modernes, XXXIII, $\mathrm{n}^{\circ} 378$, janvier 1978, p. 993-1011 ; Gianni Vattimo, La società trasparente, Milano, Garzanti, 1989, trad. La société transparente, Paris, Desclée de Brouwer, 1990.

37. Gérard Genette, Mimologiques : voyage en Cratylie, Paris, Seuil, 1976.

38. Tibor Papp / Pierre Pica, Transparence et opacité. Littérature et science cognitives, hommage à Mitsou Ronat, Paris, Éditions du Cerf, 1988.

39. Octavio Paz, Deux transparents, Marcel Duchanp et Lévi-Strauss, Paris, Gallimard, 1970.

40. Dick Higgins, Horizons. The Poetics and Theory of Intermedia, Carbondale, South Illinois Univ. Press, 1984, p. 64-65, dénonce la transparence de la langue derrière la parole.

41. Emile Zola, « Les Réalistes du Salon » (1866), in Mes haines, Genève, Slatkine, 1979, p. 307.

42. Arthur Schnitzler, Aphorismen und Betrachtungen, Frankfurt, Fischer, 1967, trad. La transparence impossible. Aphorismes, Paris, Rivages, 1990.

43. Transparence et secret, in Les Pouvoirs. Revue française d'études constitutionnelles et politiques, $\mathrm{n}^{\circ}$ 97, Paris, Seuil, 2001. Voir aussi E. Alloa, cité note 14.

44. Un exemple parmi d'autres: Marie-José Mondzain, Transparence, opacité? 14 artistes contemporains chinois, Paris, Cercle d'art, 1999.

45. André Breton, Nadja (1929), in Cuvres complètes, Paris, Gallimard (Pléiade), I, 1988, p. 651.

46. "Transparence is the highest, most liberating value in art - and criticism - today. Transparence means experiencing the luminousness of the thing itself." Susan Sontag, Against Interpretation (1966), London, Vintage, 1994, p. 13.

47. Erwin Panofsky, Die Perspektive als symbolische Form (1925), trad. La perspective comme forme symbolique, Paris, Minuit, 1975.

48. Philippe Junod, Transparence et opacité: essai sur les fondements théoriques de l'art moderne, Lausanne, L’Âge d'homme, 1976, rééd. Nîmes, Chambon, 2004.

49. Louis Marin, Opacité de la peinture. Essais sur la représentation au Quattrocento, Paris, Usher, 1989.

50. Rosalind Krauss, The Originality of the Avant-garde and Other Myths, Cambridge, MIT, 1986, p. 161.

51. Jeff Wall, Essais et entretiens 1884-2001, Paris, Ensba, 2001, p.63. Voir aussi Transparencies, London, Institute of Contemporary Arts, 1984.

52. Lucy Lippard, The Dematerialization of Art, London, Studio Vista, 1973; R. Stanislawski / Ch. Brockhaus, "Synthese der Künste und Entmaterialisierung", in Europa Europa. Das Jahrhundert der Avant-garde in Mittel- und Osteuropa, Bonn, Kunsthalle, 1994, vol. III, p. 80-121.

53. Frantisek Kupka, La création dans les arts plastiques [1922], Paris, Cercle d'art, 1989, p. 229. 


\section{RÉSUMÉS}

Loin d'être transparent, le terme "transparence» témoigne d'une polysémie source de nombreuses ambiguïtés. C'est ainsi que de son sens premier, optique, on est passé à divers emplois métaphoriques qui relèvent de domaines aussi différents que l'esthétique, la morale ou la politique. Affecté d'une connotation résolument valorisante, le concept connaît aujourd'hui une inflation qui n'est pas sans engendrer quelques malentendus.

\section{INDEX}

Mots-clés : architecture, esthétique, modernité, peinture, photographie, sculpture

\section{AUTEUR}

\section{PHILIPPE JUNOD}

Philippe Junod (1938) a enseigné l'histoire de l'art à l'université de Lausanne de 1971 à 2003. Auteur de Transparence et opacité. Essai sur les fondements théoriques de l'art moderne (1976, rééd. 2004), La musique vue par les peintres (1988), Critiques d'art de Suisse romande (1993), De l'archet au pinceau (1996), Contrepoints. Dialogues entre musique et peinture (2006), Chemins de traverse. Essais sur l'histoire des arts (2007), ainsi que divers articles sur l'histoire des jardins, l'iconographie du temps, ou l'histoire des théories artistiques.Philippe.junod@unil.ch 